\title{
Zeitlichkeit, Anachronismus und Anachronien Gegenwart und Transformationen der Geschlechtergeschichte aus geschichtstheoretischer Perspektive
}

\author{
Caroline Arni
}

Die Geschichte ist Gegenstand einer Konstruktion, deren Ort nicht die homogene und leere Zeit, sondern die von der Jetztzeit erfïllte bildet. ${ }^{1}$

\section{Ratlosigkeit}

Die unter Geschlechterhistorikerinnen immer wieder neu verhandelte Frage, ob theoretisch Avantgardistisches in der Luft liege, irgendwo Quellen von bot topics sprudelten und es so etwas gäbe wie neue Herausforderungen an die Geschlechtergeschichte, evoziert gegenwärtig eine gewisse Ratlosigkeit. Diese Ratlosigkeit kann verschiedene Gründe haben, über die ich ohne Anspruch auf Vollständigkeit zunächst spekulieren möchte, um sie dann in einem weiteren Schritt als mögliches Symptom eines spezifisch gearteten Gegenwartsbezugs zu lesen. Dazu werde ich mich mit den Begriffen des Anachronismus und der Anachronien sowie den damit verbundenen Zeitkonzepten auseinandersetzen. Darstellungen zu Entstehung, Entwicklung und Status quo der Geschlechtergeschichte tun in der Regel einen triangulären Thematisierungszusammenhang auf, der das Verhältnis zur Frauenbewegung, Kontakte mit anderen Disziplinen sowie die Position in der eigenen geschichtswissenschaftlichen Disziplin einbezieht. Wenn ich mich hier auf

I Walter Benjamin, Uber den Begriff der Geschichte [1940], in: ders., Illuminationen. Ausgewählte Schriften 1, Frankfurt a. M. 1977, 258. Ich danke den Gutachterinnen dieses Heftes furr ihre sorgfältigen Kommentare sowie der Herausgeberinnenschaft von "LHOMME. Z. F. G.“ und den Teilnehmerinnen und Teilnehmern des Kolloquiums Geschlechtergeschichte an der Universität Basel die Vortragsversionen dieses Texrs mit mir diskuriert haben. Ganz besonders danke ich Susanna Burghartz, Urs Hafner und Martin Schaffner für ihre Bereirschaft, sich auf die Lektüre eines entstehenden Textes mit seinen gedanklichen Wucherungen einzulassen. Ihre präzisen und pointierten Kommentare waren mir ebenso wohlwollende wie herausfordernde Wegweiser bei der Arbeit. 
den dritten Aspekt konzentriere, so geht es mir nicht um das Verhältnis zur Disziplin im Sinne des Einklagens der Relevanz von geschlechtergeschichtlicher Forschung für die so genannt „Allgemeine Geschichte". Stattdessen möchte ich argumentieren, dass eine Reflexion auf den Gegenwartsbezug der Geschlechtergeschichtsschreibung zwangsläufig eine Auseinandersetzung mit geschichtstheoretischen und also disziplinären Fragen nach Vorstellungen von Zeit nötig macht.

Woher also die Ratlosigkeit? Eine erste mögliche Antwort lautet, dass die Frage falsch gestellt ist. Der Brennpunkt im Feld der Geschlechtergeschichte, so lässt sich argumentieren, liegt gegenwärtig nicht in "neuen Herausforderungen“, vielmehr steht nach Jahrzehnten der methodologischen und theoretischen Debatten und Konstituierungsleistungen des Feldes schlicht empirische Arbeit an: die Fragen sind formuliert, die Gegenstände konzeptualisiert und das Handwerkszeug steht zur Verfügung.

Zweitens liegt die Vermutung nahe, dass die geschlechtergeschichtliche Forschung so breit und vielfältig geworden ist, dass es keine Herausforderungen an ,die' Geschlechtergeschichte mehr geben kann. Deren interne Heterogenität verbietet schlicht das Formulieren von übergreifenden Problemstellungen. Was nicht gleichzeitig bedeutet, dass die Vorstellung von einer Einheit des Feldes verabschiedet werden muss - jedenfalls so lange nicht, wie ein gegenständlicher Kern (Geschlechterverhältnisse und Geschlechterdifferenz) in dem Sinne trägt, als dass er die zentrifugalen und zentripetalen Kräfte des Feldes in einem Gleichgewicht hält. ${ }^{2}$ Mir dem Befund der Heterogenität lässt sich auch der immer mal wieder formulierte Eindruck widerlegen, ,die 'Geschlechtergeschichte sei an einem toten Punkt angekommen, an dem sich nichts bewegt, oder aber in einem toten Winkel, aus dem Bewegung nicht in Sicht gerät. Ausdifferenzierung heißt, dass geschlechtergeschichtliche Perspektiven diffundieren und sich spezialisieren, dass sie also vermehrt in anders definierte geschichtswissenschaftliche Felder Eingang finden, und dass mehr und enger definierte Gelegenheiten zur Präsentation von Forschungsarbeiten zur Verfügung stehen. Schließlich ist in diesem Zusammenhang auch zu beobachten, dass Geschlechterforschung zur Zeit - nicht zuletzt als Effekt von wissenschaftspolitischen Strategien - stark im interdisziplinären Zuschnitt betrieben wird, weswegen spezifisch disziplinäre Veranstaltungen in den Hintergrund getreten sind.

$\mathrm{Zu}$ bedenken ist drittens, dass Herausforderungen, die sich der Geschlechtergeschichte neu stellen, solche sind, die nicht spezifisch geschlechtergeschichtlich sind, sondern auch andere geschichtswissenschaftliche Felder konfrontieren - wie etwa das Postulat transnationaler Geschichtsschreibung und der Überwindung des Ethnozen-

2 Dann wäre in der Geschlechtergeschichte eine ähnliche Strukturlogik am Werk, wie sie die Entstehung der humanwissenschaftlichen Disziplinen prägte: Diese haben sich entlang der Bestimmung von Geenta von Gegenstandszuständigkeiten herausgebildet (die Ökonomie und der Markt, die Psychologie und das Individuum, die Soziologie und die Gesellschaft), die auch dann noch eine Einheit des Feldes stiften, wenn die epistemologischen Differenzen innerhalb der Disziplinen größer sind als jene zwischen den Disziplinen. trismus. Das bedeutet selbstverständlich nicht, dass genau diese Herausforderungen nicht auch geschlechtergeschichtlich zu formulieren sind.

Viertens schließlich lässt sich argumentieren, dass es zwar reichlich Herausforderungen gibt, dass diese aber nicht neu sind, sondern die Aufgabe vielmehr darin besteht, sich an Unerledigtem abzuarbeiten. Dann müsste man über die Erneuerung von Herausforderungen sprechen, über Versäumnisse und Aufgeschobenes nachdenken. Das scheint mir etwa bei der Diskussion um die „poststrukturalistische Wende" oder den linguistic turn der Fall zu sein, die einen Brennpunkt in der Debatte um Diskurs und Erfahrung gefunden hat. Ich rubriziere diese deshalb unter dem "Unerledigten“, weil mir scheint, dass gegenwärtig - aus ablehnender Position - eher die sie prägenden Missverständnisse oder - in apologetischer Haltung - ein zur Orthodoxie erstarrtes Mantra in die Traditionsbildung der Geschlechtergeschichte eingehen, weniger aber eine eigentliche Analyse dieser Auseinandersetzung, die künftige Arbeit zu orientieren vermag. ${ }^{3}$ Es lässt sich beim Stichwort „Unerledigtes" auch an weniger konfliktiv Befrachtetes denken, an schon seit längerem bearbeitete und nicht abgeschlossene Forschungsfragen und -gegenstände, die das Potenzial haben, Metanarrative und begriffliche Instrumentarien zu transformieren, und die ich in diesem Sinn als Herausforderungen begreifen möchte; etwa die „intersectionality" oder spezifischere Fragen wie die nach dem „Zugang von Frauen zur Sphäre des Politischen vor der Genese moderner Demokratien ". ${ }^{4}$ Begriffe wie „Gleichheit", „Ungleichheit", „Macht", „Politik“, die zugleich analytische und historische Kategorien sind, geraten als analytische in Bewegung, wenn sie historisch weiter befragt werden; in diesem Sinn stellt eine Rekonstruktion ihrer Genese eine bleibende Herausforderung dar.

Wissenschaftssoziologisch lassen sich die vier hier als Gründe für die Ratlosigkeit aufgeführten Phänomene als Ausdruck und Effekte von Etablierungs- oder Institutionalisierungsprozessen entschlüsseln, durch die sich ein Forschungsfeld zwangsläufig ausdifferenziert (die Heterogenität) und normalisiert (Empirie und die Arbeit an unabschließbaren Forschungsproblemen sind angesagt, die Herausforderungen sind ,allgemeine'). Auch wenn eine solche Analyse die Ratlosigkeit begründen und dadurch so manches Unbehagen an ihr als unbegründet ausweisen dürfte, so kann sie doch deshalb noch nicht genügen, weil solches Unbehagen ernst genommen werden muss: von den Akteurinnen in seinem produktiven Potenzial als Moment der Offenheit und aus reflexiver Perspektive als Frage nach einem Weiteren, das sich darin dokumentiert.

3 Auch die in ihrem Selbstverständnis vermittelnden Interventionen begnügen sich häufig damit, die in falschen Entgegensetzungen geöffneten Dichotomien als gleichberechtigt zu behaupten - Diskurs falschen Entgegensetzungen geöffneten Dichotomien als gleichberechtigt zu behaupten - Diskurs
und Erfahrung -, statt Genese und „stakes“ der Debarte zu analysieren und systematisch nach konzeptuellen Zusammenhängen der dabei einander entgegen geserzten Karegorien zu fragen. Schon vor längerem hat etwa Lynn Hunt eine solche Analyse begonnen: Psychoanalysis, the Self, and Historical Interpretation, in: Common Knowledge, 6, 2 (1997), 10-19.

4 Claudia Opitz, Um-Ordnungen der Geschlechter. Einführung in die Geschlechrergeschichte, Tübingen 2005, 212 . 
Dieses Weitere aber ist Ausdruck eines ambivalenten Verhältnisses zur Ausdifferenzierung und Normalisierung des Feldes und ist außerdem verknüpft mit dem „Neuen “ an den neuen Herausforderungen - und das heißt: mit Gegenwart.

Es ist ebenso trivial wie wichtig, daran zu erinnern, dass die Vergangenheit oder präziser: das Vergangene - die Materie, der Stoff der Geschichte - sich nicht ändert: Die Toten sind tot. Nun lässt sich argumentieren, dass nach dem Tod die Verwesung einsetzt, ein Zerfallsprozess, der aber immer noch ein Prozess in der Zeit ist, der die Historikerin in eine sich unablässig vergrößernde temporale Distanz zum Gegenstand setzt. Die vergehende Zeit, schreibt Reinhart Koselleck, hat eine „erfahrungsstiftende Qualität“, die „den Stellenwert vergangener Ereignisse und damit die geschichtliche Qualität dieser Ereignisse selbst" verändert. ${ }^{5}$ Ganz ähnlich sieht Paul Ricoeur in der zeitlichen Distanz zwischen der Historikerin und dem Vergangenen „nicht ein totes Intervall, sondern eine sinngenerierende Transmission " ${ }^{6}$ "Historisch", argumentiert Georg Simmel, ist ein „Inhalt" dann, wenn er zugleich ,in der Zeit ist“ und „verstanden wird“, wenn er also durch das Verstehen - die Generierung von Sinn - „verzeitlicht “ wird. ${ }^{7}$ Wandelt sich also das Vergangene durch diesen Prozess nicht, so wohl aber seine Geschichtlichkeit: Es wird zu Vergangenheit. Und diese Transformation folgt nicht dem Flussbett der chronologischen Zeit. „Sobald gefühlsmäßige Resonanzen ins Spiel kommen", schreibt Marc Bloch, „lässt sich die Grenze zwischen dem Aktuellen und dem Unzeitgemäßen nicht mehr unbedingt mit dem mathematisierenden Messband des Zeitintervalls bestimmen ". ${ }^{8}$ Emotionalen Widerhall aber erzeugt das Vergangene nur dort, wo sich an ihm Neugier entzündet.

Die Toten also sind tot und das Vergangene ändert sich nicht. Was sich verändert, im erfabrungsstiftenden und sinngenerierenden Vergehen der Zeit, das ist die Geschichtlichkeit des Vergangenen. Und es ist die Neugier, die diese Transformation gestaltet, denn Geschichte existiert nur, so Paul Veyne, „im Verhältnis zu den Fragen, die wir an sie richten". ' Die Fragen aber kommen aus der Gegenwart, dieser, wie Nicole Loraux schreibt, „effizientesten Antriebskraft des Verstehenwollens". ${ }^{10}$ Also müsste „das Neue" in der Gegenwart liegen, in der die Fragen gestellt werden. Wenn es denn so wäre, dass sich der Geschlechtergeschichte keine neuen Herausforderungen stellten, dann deshalb, weil es für sie keine "neue" Gegenwart gäbe. Das trifft jedoch schon deshalb nicht zu, weil, wie Susanna Burghartz argumentiert, auch „Kontinuität Ergebnis historischer Pro5 Reinhart Koselleck, Vergangene Zukunft. Zur Semantik geschichtlicher Zeiten, Frankfurt a. M. 1989,
10.

6 Paul Ricoeur, Temps et Récit, Bd. 3: Le temps raconté, Paris 1985, 3996.

7 Georg Simmel, Das Problem der historischen Zeit, Berlin 1916; 12.

8 Marc Bloch, Apologie pour l'histoire ou Métier d'historien, Paris 1997 (Orig. 1949), 59.

9 Paul Veyne, Die Originalität des Unbekannten. Für eine andere Geschichtsschreibung, Frankfurt a. M. 1988 (Orig. 1976), 8.

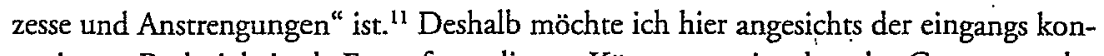
statierten Ratlosigkeit als Frage formulieren: Könnte es sein, dass der Gegenwart der Geschlechtergeschichte heute wohl ein erkenntnisrelevantes Moment zukommt, dass aber dieses Moment nicht eines des Antriebs ist, sondern eines der Lähmung? Damit aber stellt sich zunächst die Frage: Was ist die Gegenwart der Geschlechtergeschichte?

\section{Feministische Gegenwart}

Zwar hat sich die Geschlechtergeschichte seit ihren Anfängen zunehmend den Anforderungen tagespolitischer Programmatik entbunden, wovon nicht zuletzt die rasche und erfolgreiche Etablierung des Begriffs "Geschlechtergeschichte" und der Bedeutungsverlust des Begriffs „Feministische Geschichtswissenschaft" zeugen. Allerdings sei hier auch registriert, dass solch tagespolitische Anforderungen gegenwärtig transformiert und nun nicht mehr im Kontext der feministischen Bewegung, sondern im Rahmen institutionalisierter Gleichstellungspolitik sowie einer ökonomisierten Logik der Forschungsförderung wiederkehren: als Ruf nach der Bereitstellung von unmittelbar verwertbarem Wissen oder, genauer, von Expertisen. Auch wenn also die Geschlechtergeschichte sich den Anforderungen der tagespolitischen Programmatik einer sozialen Bewegung entbunden hat und nicht mehr als „(direkte oder indirekte) Handlungsanleitung für die Frauenbewegung " dienen kann/will/soll, ${ }^{12}$ so ist sie in der für sie formativen Gegenstandskonstituierung und - das ist wichtig - im gegenwärtigen zeitgeschichtlichen Kontext in einem sehr grundsätzlichen und nicht programmatischen Sinn noch immer feministische Wissenschaft. Sie ist es insofern, als sie sich die Erforschung von Geschlechterverhältnissen und Geschlechterdifferenz als historische Sachverhalte und, umgekehrt, als geschichtsmächtige soziale Tatsachen zum Anliegen macht und dies als Geschichtswissenschaft tut, das heißt: bemüht um eine Analyse des historisch kontingenten Gewordenseins der Verhältnisse, was immer auch ein prinzipieller Nachweis von Veränderbarkeit ist. So verstandene Geschichtsschreibung ist Kritik (in diesem Fall an naturalisierenden Ideologien), und Kritik - darauf hat jüngst Joan Scott im dezidierten Rückgriff auf Gewährsmänner von Karl Marx über Theodor W. Adorno und Max Horkheimer bis zu Michel Foucault hingewiesen ${ }^{13}$ - ist eine Passion, ein Gefühl also, das sich aus der ebenso lustvollen, wie Schwindel erregenden Erkenntnis nährt (die man auch eine intellektuelle Erfahrung nennen könnte), dass die Dinge nicht sein müssen, wie sie sind.

II Susanna Burghartz, Wandel durch Kontinuität? Zur Moralpolitik von Reformation und Konfessionalisierung, in: traverse, $7,1(2000), 23-35,32$

12 Opitz, Um-Ordnungen, wie Anm. 4, 155.

13 Vil. den Vortrag von Joan Scott auf der "European Social Science Histary Conference" 2006 (Joan W. Scott, History-writing as Critique, in: Keith Jenkins u. a. Hg., Manifestos for History, London, erscheint 2007). 
In diesem gefiihlsmäßigen Resonanzraum bewegt sich die Geschlechtergeschichte noch immer - und zwar gleichsam nolens volens in einer Gegenwart, in welcher der Feminismus als soziale Bewegung gern für beendet (oder gar gescheitert) erklärt wird, während zugleich die ihn begründenden Anliegen - die Forderung nach Geschlechtergleichheit und die Kritik an Geschlechterungleichheit - mediale und politische Effekte en masse zeitigen. Deren Gestalten freilich sind nicht so einfach zu bestimmen. Erstens, weil sich jede zeitdiagnostische Anstrengung aus feministischer Perspektive verstörenden Widersprüchen gegenüber sieht, wie etwa demjenigen zwischen formalrechtlicher Gleichstellung und praktischer Ungleichstellung in politischen und wirtschaftlichen Belangen. Zweitens, weil die Zirkulation feministischer Anliegen nicht minder verstörende Perversionen möglich macht, wie etwa die, dass ganze Kriege rhetorisch im Namen der Rechte von Frauen geführt werden, wenn und solange das geopolitischen Zwecken dient. Oder die, dass einem eigentümlichen Sprechzwang unterliegende Politiker die moralische ,Höherwertigkeit' so genannt christlich-abendländischer Kultur vorzugsweise mit dem dieser angeblich immer schon innewohnenden "Wert" der Geschlechtergleichheit begründen (als hätte das Gleichheitspostulat in Sachen Geschlechter nicht gegen kirchlich-religiöse Deutungsmacht, gegen den Patriarchalismus „Alteuropas “ und schließlich auch gegen die Ausschlussoperationen der europäischen politischen Moderne durchgesetzt werden müssen). Drittens gesellt sich zu diesen politischen und medialen Phänomenen das, was in den Augen einer Historikerin ein ganzes Kuriositätenkabinett an gespensterhaften Wiedergängern (auch Zombies oder Untote) ist: wenn etwa die Naturalisierungsanstrengungen in Sachen Geschlechterdifferenz und -verhältnisse aus dem 19. Jahrhundert in Gestalt der Evolutionary Psychology in Wissenschaftsjournalismus, Forschungsförderung und auf dem Buchmarkt Urstände feiern, oder wenn Feuilletonisten sich besonders klug dünken, weil sie auf die in gut hundertfünfzig Jahren schwer angegraute Idee verfallen, generative Weiblichkeit als sozialen Kitt anzupreisen, der die allzu locker gewordenen Fugen einer wieder einmal anomischen Gesellschaft schließen soll.

Wer angesichts dieser Konstellation in zeitdiagnostischer Absicht nach Erfolg oder Scheitern des Feminismus gefragt wird, tut gut daran, keine Antwort zu geben, sondern entweder im Kant'schen Sinne ein Paradox ,ins Publikum zu spielen" "14 - dass beides der Fall ist oder keins von beidem - oder aber theoretisch gewitzt und historisch gelassen die Frage als eine falsch gestellte oder doch zumindest verfrühte zurückzugeben. Indes werfen die geschilderten Phänomene der Widersprüche, der Perversionen und der Wiedergänger gerade deshalb sehr wohl Fragen auf, weil sie nicht unabhängig von Diskurs und Bewegung des Feminismus gedacht werden können und so im feministischen oder auch einfach kritisch-analytischen Bewusstsein gefïhlsmäßige Resonanzen erzeugen -

I4 Immanuel Kant, Anthropologie, erster Teil, anthropologische Didaktik, in: Werkausgabe, Bd. XII, hg von Wilhelm Weischedel, Frankfurt a. M. 1964, 410 deren eine, so mein Argument, die bereits aufgeworfene Lähmung ist. Diese Fragen aber sind genuin geschlechterbistorische Fragen, weil sie etwas mit Temporalität, mit dem Denken der Zeit und ihrer Rhythmen zu tun haben.

\section{Kritik und Gebrauchsweisen des Anachronismus}

Die Frage nach dem Verhältnis der Geschichte zur Gegenwart im Sinne eines von Historikerinnen-bewohnten und bearbeiteten Verhältnisses wirft, wie Nicole Loraux schreibt, immer schon das „gefürchtete Problem des Anachronismus in der Geschichtsschreibung" (le redoutable problème de l'anacbronisme en histoire) auf. ${ }^{15}$ Anachronismus bedeutet sinngemäß das chronologische Verlegen von Personen, Ereignissen, Objekten und Bräuchen oder spezifischer in der Geschichtswissenschaft: das Importieren von Begriffen - und den damit bezeichneten Gegenständen - in Epochen, die diese nicht gekannt haben. Gemeint ist also eine "Verfehlung gegen die Chronologie" (faute contre la chronologie), und zwar nicht hinsichtlich von Daten, sondern von Epochen (la confusion des epoques), wie Jacques Rancière schreibt, beziehungsweise hinsichtlich „der Zugehörigkeit zu einer Epoche“ im Sinne „kollektiver Ermöglichungsbedingungen subjektiver Weltsicht", wie es Jakob Tanner konzeptuell zugespitzter formuliert. ${ }^{16}$ Der über seine scharfe Verurteilung durch Lucien Febvre sozusagen paradigmatisch gewordene Fall solcher Verfehlung ist die These von Abel Lefranc, Rabelais sei, obschon Zeitgenosse des 16. Jahrhunderts, ein freidenkerischer Rationalist und damir ein heimlicher Atheist gewesen. ${ }^{17}$

Solcher Anachronismus sei, so Loraux weiter mit Bezug auf die Febvre'sche Kritik, "das schwarze Schaf der Historiker, die Kapitalsünde gegen die Methode, und allein der Name ,Anachronismus' bedeutet eine ehrentührige Anklage, die Anklage - alles in allem - nicht historisch vorzugehen, sondern sich der Zeit und der Zeiten in einer irreführenden Weise zu bemächtigen“. ${ }^{18}$ Vor dieser Anklage gelte es eine „kontrollierte Praxis des Anachronismus" (une pratique contrôlée de l'anachronisme) zu verteidigen: als ein bewusst eingegangenes Risiko, den Zeitgenossen Fragen zu stellen, die diese sich selbst nicht gestellt haben. ${ }^{19}$

I5 Loraux, Eloge, wie Anm. 10, 23.

16 Jacques Rancière, Le concepr d'anachronisme et la vérité de l'historien, in: L'Inactuel, 6, 1996, 53-68, 54f; Jakob Tanner, Historische Anthropologie zur Einführung, Hamburg 2004, 68. Vgl. Rancière, ebd., 54 fauch zu weitergehenden lexikalischen Erörterungen sowie zur Herkunft des Begriffs aus der Poetik. Zu ciner lo ischen Kritik am Begriff vel. Annerre Barnes u. Jonatan Barnes, Time Out of Joint: Some Reflections on Anachronism, in: The Journal of Aesthetics and Art Criticism, 47, 3 (1989), 253-261.

17 Lucien Febvre, Le problème de l'incroyance au XVe siècle. La religion de Rabelais, Paris 1968

18 Loraux, Eloge, wie Anm. 10, 23.

I9 Loraux, Eloge, wie Anm. 10, 28 
In verschiedenen jüngeren Lekrüren des Anachronismusvorwurfs lassen sich drei Thematisierungs- oder Gebrauchsweisen des Phänomens ausmachen. Alle gründen auf demselben Verständnis von Anachronismus im oben beschriebenen Sinn, legen aber den Schwerpunkt auf unterschiedliche Implikationen und konzeptionelle Fluchtpunkte. Diese drei Gebrauchsweisen möchte ich im Folgenden kurz ausfiuhren; dabe wird sich allerdings zeigen, dass die zweite Gebrauchsweise eher eine Analyse ist, die als Begründung der dritten fungiert, während diese ihrerseits den Begriff des Anachronismus als sinnlos ausweist und zugunsten desjenigen der Anachronie hinter sich lässt.

Die erste Gebrauchweise ist in den zitierten Ausführungen von Nicole Loraux bereits deutlich geworden: Es handelt sich um den Gedanken, dass anachronistisches Denken dann für die historiographische Arbeit einträglich ist, wenn bewrusst aus der Aktualität Fragen gewonnen werden, die im Sinne Marc Blochs - und auf ihn bezieht sich Loraux explizit - ein Wechselspiel eröffnen, auf dessen Feld die Vergangenheit und die Gegenwart auseinander erschlossen und verstanden werden. Kontrollierter Anachronismus in diesem Sinn ist eine „Methode, die darin besteht, sich mit Fragen der Gegenwart an die Vergangenheit zu richten, um ausgestattet mit dem, was man an der Vergangenheit verstanden hat, wieder zur Gegenwart zurückzukehren ". ${ }^{20}$ Loraux konkretisiert das am Beispiel der „öffentlichen Meinung", ein Begriff und Phänomen der Gegenwart, das sie in der Antike untersucht. Dabei geht es nicht darum, das Gleiche (des Gegenstands) im Andern (der Vergangenheit) wieder zu finden: „es muss gelingen, hin und her zu gehen und seinen Standort immer wieder zu verschieben, um so zu den nötigen Unterscheidungen zu gelangen". ${ }^{21} \mathrm{Es}$ wird also zu heuristischen Zwecken von der Gegenwart ausgehend ein übergreifender Gegenstand konstruiert, um dessen Transformationen respektive historische Differenzen sichtbar zu machen.

In einer radikaleren, wahlweise poststrukturalistisch oder wissenssoziologisch begründbaren Variante dieser Gebrauchsweise könnte man auch formulieren, dass solcher Anachronismus nicht nur eine bewusst gewählte Heuristik zur Gewinnung von Fragen, sondern recht eigentlich unvermeidlich ist, da Erkenntnisinteressen ebenso wie die Instrumente der Analyse und Interpretation immer in historisch spezifische epistemologische Möglichkeitsbedingungen gefasst oder „,standortgebunden“" (Karl Mannheim) sind; der Standort der Historikerin aber ist unverrückbar ihre Gegenwart. Und diese Gegenwart stattet sie nicht nur kognitiv aus, sondern verstrickt sie auch emotional. In diesem Sinn lässt sich mit Sophie Wahnich festhalten, dass beim kontrollierten Anachronismus es „nicht die kollektive Erinnerung [ist], welche die Arbeit organisiert, sondern die Aktualität insofern, als sie im Historiker Affekte erzeugt". ${ }^{\text {" }}$

2o Loraux, Eloge, wie Anm. 10, 28

2I Loraux, Eloge, wie Anm. 10, 29, 33.

22. Sophie Wahnich, Sur l'anachronisme contrôlé, in: CllO. Histoire, Femmes et Saciétés \& Espaces Temps, 87-88 (2004), 140-146, 143
Einen zweiten Zugang formuliert Jacques Rancière, der den Vorwurf des Anachronismus auf die ihm zugrunde liegenden geschichtstheoretischen Annahmen untersuch und dabei besonders das mit dem Vorwurf verknüpfte Epochenkonzept behandelt Hinter dem Vorwurf verberge sich ein spezifisches Zeitkonzept, das die Anklage ers sinnhaft mache: die Vorstellung einer Zeit, die nach dem Prinzip nicht der Sukzession, sondern der Simultaneität funktioniere, indem sie historische Subjekte als solche ausweise, die ihrer Zeit ähnlich und mit dieser sozusagen "gleichzeitig" seien (contemporanéité), und so homogene epochale Einheiten stifte. Gegenwart sei in diesem Zeitkonzept „reine Gegenwart" (pur présent), gereinigt von andern Zeiten, und ein Subjekt, das weltanschaulich aus dieser Zeit ausbreche, nicht denkbar beziehungsweise historisch falsch gedeutet, also ,unwahr ${ }^{.23}$ So bringe sich im Vorwurf des Anachronismus ein spezifisches Regime der historiographischen Wahrheitsproduktion zur Geltung, das de Geschichtsschreibung ihre Wissenschaftlichkeit und Identität garantieren solle: Existenz in der Vergangenheit gibt es nur als Kopräsenz mit der vom Prinzip der Gleichzeitigkeir erzeugten und von der Historiographie als solche erkennbaren Epoche.

Dieses Zeitkonzept, so Rancière weiter, verleibe sich mit der Vorstellung einer reinen Gegenwart zugleich die Idee der Ewigkeit ein: Es negiere die fließende Zeit und bewahre so das Gegenteil von Geschichtlichkeit in sich auf. Folglich sei der Vorwurf des Anachronismus „pervers", da das Zeitkonzept, das ihn sinnhaft mache, nicht historisch sei: „Das Konzept des ,Anachronismus' ist anti-historisch, weil es die Bedingungen von Historizität verbirgt. Es gibt Geschichte gerade insofern, als Menschen nicht ihrer Zei ,ähneln', insofern als ihr Handeln mit, ihrer' Zeit bricht, mit der temporalen Linie, die sie an ihren Ort verweist, indem sie ihnen aufträgt, von ihrer Zeit einen bestimmten ,Gebrauch' zu machen. Aber dieser Bruch ist selbst nur deshalb möglich, weil diese Linie der Temporalität mit andern verbunden werden kann, weil in ,einer ' Zeit eine Vielzahl von temporalen Linien gegenwärtig ist. "24 Statt von Anachronismus als einer fehlerhaften und fehlbaren Operation des Historikers zu sprechen, muisse man sich deshalb mit „Anachronien" beschäftigen: ,gegenzeitliche "Ereignisse, Begriffe, Bedeutungen die ihre Zeit gegen den Strich bürsten, die Sinn in einer Weise zirkulieren lassen, die jeglicher Kozeitlichkeit, jeder Identität einer Zeit mit ,sich selbst' entwischt ${ }^{\text {“ }}{ }^{25}$ Damit

23 Rancière, Concept, wie Anm. 16, 60. Auf der Grundlage dieses Zeitkonzepts ist dann eben kein atheistischer Rabelais denkbar.

24 Rancière, Concept, wie Anm. 16, 65f, Zitat 66

25 Rancière, Concept, wie Anm. 16, 67. Die Begrifflichkeit ist insofern etwas verwirrlich, als Rancière in seinem Text von 1996 von "anachronie" spricht, Loraux dagegen 1993 in explizitem Bezug auf Rancière von „achronie" (Loraux, Eloge, wie Anm. 10, 22). Möglicherweise lässt sich die Differen damit erklären, dass Loraux sich auf einen mündlichen Vortrag von Rancière von 1991/92 beziehr: oder aber sie ändert die Begrifflichkeit in begründeter Korrektur, da die griechische Präposition „ana-“ für "hindurch" steht, das griechische Negationselement "a-" " für "gegen". Beide Begriffe werden verwendet; in der Erzähltheorie etwa gilt Achronie (nicht rekonstruierbare Chronologie) als ein Extremfall der Anachronie (die Abfolge der Erzählung fällt nichr zusammen mit der chronologischen Abfolge der ezzählten Ereignisse). 
verschiebt Rancière die Diskussion von einer - in seinen Augen vermeintlichen - Problematik des Vorgehens zu einem Sachverhalt: Anachronismus gibt es nicht, wohl aber Anachronien, und um nichts weniger geht es bei diesen als um die „Macht, Geschichte $\mathrm{zu}$, machen "c (un pouvoir de 'faire' l'bistoire). ${ }^{26}$

Hier kommt das ins Spiel, was ich die dritte Gebrauchsweise des Anachronismus nennen möchte: Anachronismus, respektive jetzt Anachronien, als ein Zugriff auf Mechanismen des Wandels beziehungsweise als eine auf Zeitlichkeit bezogene, nicht deterministische und akteurtheoretische Konzeption von Wandel durch Handlungsmacht (im Sinn von agency). Im Zeitkonzept, das gemäß Rancière im Vorwurf des Anachronismus - ja überhaupt in der Vorstellung, es gäbe erwas, das sinnhafterweise Anachronismus zu nennen wäre - operiert, gibt es keine "Gleichzeitigkeit des Ungleichzeitigen ".27 Genau darin aber besteht das implizite Konzept von Wandel und Handlungsmacht, das Rancière in den Begriff der „Anachronie“ schlägt und das Jakob Tanner in seiner Rancière-Lektüre explizit als solches auf den Punkr bringt. Wandel komme dann in Gang, "wenn Menschen sich der Zumutung widersetzen, mit der Zeit, in der sie leben ,ähnlich' zu werden “. ${ }^{28}$ Die Debatte über den Anachronismus interpretiert Tanner entsprechend als eine Auseinandersetzung über die Konzeptualisierung von Handlungsmöglichkeiten: Steht auf der Seite des Anachronismusvorwurfs und der Vorstellung geschlossener Epochen reiner Gegenwart die Annahme einer „homogenen Verbindung von (objektiver) Struktur und (subjektiver) Mentalitär“, die ein „deterministisches Handlungsmodell“ begründet, so wird dem auf der andern Seite die Möglichkeit eines Handelns wider die Struktur (a rebours) beziehungsweise in "Spielräumen entgegengehalten. ${ }^{29}$ Ersteres Modell kann Wandel nicht konzipieren. In zweiterem ergibt sich die Möglichkeit von Wandel aus der gleichzeitigen Verfügbarkeit über sich widersprechende Bedingungen, Orientierungen und Ressourcen des Handelns (Sinnstrukturen, materiale Bedingungen etc.), die gedacht werden - und hier ist der springende Punkt - als solche, die verschiedenen Epochen angehören beziehungsweise deren Widersprüchlichkeit ihrer differenten zeitlichen Zugehörigkeit zuzuschreiben ist. Nur so macht der Begriff der "Anachronie“ Sinn, der ja statt der Einheitlichkeit einer Epoche deren Aufsplitterung in verschiedene temporale Elemente oder Linien annimmt, die eben "gegen" die Epoche sich bewegen. Ein Beispiel dafür formuliert hübsch Siegfried Kracauer: „so gehörten die überladenen Intérieurs der zweiten Hälfte des

26 Rancière, Concept, wie Anm. 16, 68.

27 Ich gehe hier nicht ein auf die verästelte Genealogie dieser berühmten Formel, deren Urheberschaft sinngemäß oder wördlich Wilhelm Pinder, Marc Bloch und/oder Karl Mannheim zugeschrieben wird. 28 Tanner, Anthropologie, wie Anm. 16,69.

29 Tanner, Anthropologie, wie Anm. 16, 69f. Wobei man bei ersterem Modell zusärzlich nicht nur die homogene Verbindung voraussetzen müssse, sondern ebenso die interne Homogenität von jeweiligen strukturellen und mentalen Konstellarionen. Die Zuordnung des handlungsdeterministischen Modells zu historiographischen Strömungen oder schon nur ihrer Vertreter gestaltet sich schwierig so weist Tanner darauf hin, dass der Anachronismus-Kritiker Lucien Febure selbst mit der Widersprüchlichkeit von Normen und Institutionen einer Epoche" argumentierte; vgl. ebd., 69. neunzehnten Jahrhunderts der gleichen Epoche an wie die in ihnen entstandene Gedanken und waren dennoch nicht ihre Zeitgenossen". ${ }^{30}$

Widersprüchlichkeit, die Wandel ermöglicht und erzeugt, ist in diesem Konzept also immer schon eine in der Zeit, während die Möglichkeit einer Widersprüchlichkeit im Gleichzeitigen gewissermaßen übersprungen wird. Da dieser Schritt in den oben referierten Texten nicht explizit gemacht wird, muss ungeklärt bleiben, ob sich dahinter eine - durchaus plausible - spezifisch historiographische, nämlich temporaltheoretische Fassung von zunächst nicht zwingend in Gestalt der „Ungleichzeitigkeit des Gleichzeitigen" gedachten Konzeptionen von Dynamik durch widersprüchliche Konstellationen verbirgt: $Z u$ denken ist hier an Wandel, der angetrieben wird von Antagonismen zwischen unterschiedlichen Dimensionen des Sozialen (zum Beispiel zwischen ökonomischen Bedingungen und sozialen Verhältnissen) oder von solchen innerhalb einer Dimension (zum Beispiel in sich widersprüchliche Sinnstrukturen). ${ }^{31}$ Erst wenn die antagonistischen Elemente unterschiedlichen Zeiträumen zugewiesen werden können, wenn die Widersprüche also temporal als Ungleichzeitigkeiten gedeutet werden, hat man es mit Anachronien zu tun. Ob diese temporale Fassung von Widersprüchen im geschilderten Modell als eine im Prinzip empirische Frage gehandelt wird, oder ob die fraglichen Widersprüche immer schon als Ungleichzeitigkeiten verstanden sind, weil sie als von unterschiedlichen Rhythmen und Dynamiken verursacht angesehen werden, wird in den konsultierten Texten nicht ausgefiihrt.

Damit verbunden ist die ebenfalls offene Frage danach, ob dieses Modell von Wandel immer schon mit einem Dominanz- oder Hegemoniekonzept einhergeht. Verweist das vorgreifend Anachronische einer spezifischen Epoche in die Zukunft einer ihr nachfolgenden, in der es das Dominante sein wird, während es in der Vergangenheit, in der es bereits auftritt, marginal ist (wie der Atheismus des Rabelais)? Und, umgekehrt, bewahrt das rückgreifend Anachronische Überreste auf, die einer Vergangenheit angehören, in der es dominant war? Die Frage drängt sich deshalb auf, weil einerseits die Einheitlichkeit von Epochen konzeptuell aufgebrochen wird, andererseits aber von Anachronien sinnhaft nur dann gesprochen werden kann, wenn es etwas gibt, das eine Epoche so dominiert, dass es sie zu definieren vermag, und "gegen" das sich bestimmte Sachverhalte „zeitlich“ verhalten können. ${ }^{32}$ Oder müsste man stattdessen mit Siegfried

30 Siegfried Kracauer, Geschichte - Vor den letzten Dingen. Schriften, Bd. 4, Frankfurt a. M. 1971, 140. 3x Hier lässt sich auch an den von Giovanni Levi vor einiger Zeir gemachren Vorschlag denken, für die Konzeptualisierung von Handlungsspielräumen die Lücken und Widersprüche innerhalb normariver Systeme zu beachten; vgl. Giovanni Levi, On Microhistory, in: Peter Burke Hg., New Perspectives on Historical Writing, Cambridge/Oxford 1991, 93-113, 94f; vgl. von der Soziologie her: Bernard Lahire, Esquisse du programme scientifique d'une sociologie psychologique, in: Cahiers internationaux de Sociologie, 56 (1999), 29-55.

32 Dieses Konzept wäre dann schon bei Wilhelm Dilthey zu finden (zit. nach Kracauer, Geschichte, wie Anm. 30, 141): „Neben der herrschenden, großen, durchgehenden Tendenz, die der Zeit ihren Charakter gibt, bestehen andere, die sich ihr entgegenserzen." Allerdings habe Dilthey, so Kracauer, daraus noch keine Schlüsse berreffend einer Kririk der kontinuierlichen chronologischen Zeit gezogen. 
Kracauer, der sich - wenn auch in anderen Begriffen und anderer Akzentuierung - mit denselben Fragen beschäftigt hat, formale und sachliche Zeitgenossenschaft unterscheiden? Ganz ähnlich wie Rancière spricht Kracauer davon; dass die „messbare Zeit" einer Epoche (die bei ihm „Zeitraum" heißr) „aufgehoben [ist] durch die Bündel geformter Zeiten, in denen die mannigfaltigen verständlichen Ereignisreihen sich entwickeln": „Zu einem gegebenen geschichtlichen Moment sind wir dann einer Anzahl von Ereignissen konfrontiert, die wegen ihres Standortes in verschiedenen Bereichen nur im formalen Sinn gleichzeitig sind." Zugleich behält der Zeitraum seine Bedeutung, „insofern diese Bündel in bestimmten Augenblicken, die dann für sie alle gültig werden, leicht zusammenwachsen " ${ }^{33}$ Nicht die Dominanz einer temporalen Linie eines Bereichs sozialer Wirklichkeit demarkiert dann eine Epoche oder einen Zeitraum, sondern die formale Gleichzeitigkeit, die es ermöglicht, dass "divergente Zeiten ${ }^{134} \mathrm{zu}-$ einander in Zusammenhang geraten - was die immer empirische Frage eröffnet, unter welchen Bedingungen und in welchen Konstellationen dies geschieht.

An diese Überlegungen anschließend lässt sich weiter fragen: Müssten nicht all die ausgeführten Denkfiguren selbst wiederum historisiert werden $?^{35} \mathrm{Da}$ ich diese Fragen hier nicht weiter verfolgen kann, möchte ich vorerst abschließend notieren: Ein aus der Kritik am Anachronismusvorwurf gewonnenes Konzept von Wandel durch Anachronien verabschiedet das Denken in Epochen nicht, sondern konzipiert diese zunächst einmal als durchlässig und heterogen. Die Heterogenität wird dabei als eine der differenten zeitlichen Zugehörigkeit von gleichzeitig anwesenden Elementen gedacht, die Handlungs- und Veränderungsmacht historischer Subjekte begründet und damit Wandel erklärt.

\section{Radikale Historisierung und reflexiver Anachronismus in der Geschlechtergeschichte}

Seit ihren Anfängen ist die Geschlechtergeschichte eine anachronistisch verfahrende Geschichtsschreibung oder - jetzt anders formuliert - eine Form der Geschichtsschreibung, die ihre Fragen im Sinne eines kontrollierten Anachronismus aus der Gegenwart bezieht und ihr Augenmerk auf Anachronien richtet. Ich werde das in diesem und den beiden folgenden Teilen zu veranschaulichen versuchen.

33 Kracauer, Geschichte, wie Anm. 30, Zitate 146, 139f, 146

34 Kracauer, Geschichte, wie Anm. 30, 141.

Im Sinne von François Hartog fügt sich eine solche Konzeptualisierung von Wandel in das moderne nachrevolutionäre und auch nach-Benjamin'sche Historiziä̈rsregime, dem zufolge die Zukunft bzw. zunehmend die Gegenwart die Vergangenheit aufklärt, Geschichte aber nicht als eine lineare Entwicklung homogener Einheiten gedacht wird; vgl. François Hartog. Temps er hisroire $C o m$ Enécrire l'histoire de France?" in: Frienne Francois $\mathrm{H}$. Lien He Mémo modèle français à un projet allemand, Berlin 1996, 29-53.
Dass die Geschlechtergeschichte gegenwartsbezogen verfährt, gilt für konkrete thematische Forschungen wie zum Beispiel solche zur Geschichte weiblicher Intellektueller. Wenn etwa die französische Zeitschrift „CLIO" 2001 in einer Nummer zum Thema „Intellectuelles" Frauen wie Aspasia oder Christine de Pizan und aufgeklärte Salonnièren abhandelt, dann ist das ein eklatanter Fall von Anachronismus - und dass hier eine Kategorie nicht nur zeitlich, sondern außerdem substanziell verlegt wird, verdeutlicht noch den möglichen Gewinn kontrolliert anachronistischen Fragens: ${ }^{36}$ Denn Begriff und Figur des Intellektuellen kommen erst in den 1890er Jahren auf und zwar in männlicher Kodierung. ${ }^{37}$ Den Begriff kontrolliert anachronistisch auf Frauen vor dem Ende des 19. Jahrhunderts anzuwenden, erbringt insofern eine doppelte heuristische Distanzierungsleistung, die aus der Gegenwart (in der es weibliche Intellektuelle gibt, nach deren Geschichte gefragt werden kann) eine historische Fragestellung gewinnt: Welches sind die Formen und Gestalten weiblicher Äußerungs-, Artikulations- und Reflexionsmacht, die aus der Traditionsgeschichte der Intellektuellenfigur hinausdefiniert und hinaus geschrieben werden mussten, um eben diese Figur zu einer klar definierten zu machen und andere Gestalten und Akteure der Wissensproduktion in Form öffentlicher Einmischung und Kritik der Amnesie anheim fallen zu lassen? Zu welchen Antworten auch immer eine solche Unternehmung kommt, in jedem Fall werden Begriff, Gegenstand und Traditionsgeschichte des/der Intellektuellen geschärft und verfeinert daraus hervorgehen und allfällige Amnesien aufgebrochen sein. ${ }^{38}$

Dass die Geschlechtergeschichte eine anachronistische Form der Geschichtsschreibung ist, gilt auch für ihr grundlegendstes analytisches Instrumentarium, hat sie doch nicht nur ihre Erkenntnisinteressen von allem Anfang an dezidiert aus der Gegenwart bezogen - zunächst aus der Kritik an der Unterdrückung von Frauen, später der Kritik an einer naturalisierten Geschlechterdifferenz -, sondern auch die diesen Fragen entsprechenden Begriffe, Konzepte und Kategorien: „Geschlechterverhältnisse", „Geschlechterdifferenz", „Geschlechterrollen“, „Geschlechternormen“, ,Heterosexualität" und „Homosexualität", vielleicht gar „Männlichkeit" und „Weiblichkeit“, sicher aber „sex/gender“.,99

36 Vgl. CLIO. Histoire, Femmes et Sociétés, 13 (2001); aber auch bereits LHOMME. Z. F. G., 2, 2 (1991). 37 Zur Intellektuellenforschung vgl. z. B. Christophe Charle, Vordenker der Moderne. Die Intellektuellen im 19. Jahrhundert, Frankfurt a. M. 1997 u. Nicole Racine u. Michel Trebitsch Hg., Intellectuelles. Du genre en histoire des intellectuels, Paris 2004. Letzterer Titel ist bezeichnenderweise in der Reihe Histoire du temps présent" erschienen und sein Cover ziert ein Porrär der Heiligen Katharina von "Histoire du temps présent" erschienen und sein Cover ziert ein Porträr der Heiligen Ka
Alexandrien, Schuzzherrin der Philosophen, von Paolo di Giovanni Fei (ca. 1345-1411).

38 Vgl. z. B. Nicole Racine u. Michel Trebitsch, Présentation, in: dies., Inrellectuelles, wie Anm. 37, 13$40,21 f f$, die aus der Beschäftigung mit weiblichen Inrellektuellen drei alternative Chronologien der Intellektuellengeschichte gewinnen.

39 Ein besonders anachronistisches Begriffspaar, ist es doch einer Zeit zugehörig, die endlang der für sie typischen disziplinären Aufteilung von Wissen über den Menschen dessen Körperliches als Biologisches von dessen Nicht-Körperlichem als Sozialem, Kulturellem und Psychischem unterscheidet. Nicht nur die Unterscheidung, sondern auch die Kategorisierung des Unterschiedenen sind historische Setzungen. 
Mit solchen theoretisch gesättigten Begriffen, die in der Anwendung auf der Gegenwart vorangehende Zeiten anachronistisch sind, löste die Geschlechtergeschichte die Forderung Paul Veynes nach einer Geschichtsschreibung ein, „die sich nicht darauf beschränkt zu schildern, nicht einmal darauf, zu verstehen, sondern die ihren Stoff durch Rückgriff auf die Konzeptualisierung der Humanwissenschaften, auch moralische oder politische Wissenschaften genannt, strukturiert" ${ }^{\text {" }}{ }^{60}$ Freilich, das ist nichts anderes als das Postulat einer theoriegeleiteten Geschichtsschreibung, wie es in den 1970er Jahren Hochkonjunktur hatte und Resonanz primär in einer historischen Sozialwissenschaft fand, deren strukturdeterministische Verkürzungen in den 1980er Jahren aus der Perspektive kulturwissenschaftlicher Anstrengungen und einer erneuerten, nicht-historistischen Hermeneutik unter Beschuss gerieten. Nicht nur hatte die Frauen- respektive später Geschlechtergeschichte an beiden Strömungen teil, sondern sie trieb auch die Kritik voran, die von ersterer zu zweiterer überleitete. Auch wenn sie sich in diesem Kontext von ihren eigenen subsumptionslogischen Großstrukturtheorien - wie etwa den Patriarchatstheorien - verabschiedete, auch wenn sie sich bezeichnenderweise im Verlauf der 1980er Jahre nicht entlang einer Theorie, sondern via die Schärfung von Analysekategorien - sex und gender - formierte, so blieb ihr doch just so ein begriffliches Instrumentarium erhalten, das es im Sinne des kontrollierten Anachronismus erlaubte, das Risiko einzugehen, den Zeitgenossen Fragen zu stellen, die diese sich nicht gestellt haben. Und dabei kam sie vielleicht Veynes Forderung nach Konzeptualisierung näher als die großtheoriegeleiteten Historiographien der 1970er Jahre.

Allerdings ist dieses begriffliche Instrumentarium gerade wegen seines anachronistischen Gehalts und des implizierten - wenn auch kontrollierten - Anspruchs, einen bei aller Transformation überzeitlich mit sich identischen Sachverhalt zu bezeichnen, als geschichtswissenschaftliches Handwerkszeug instabil. Und zwar deshalb, weil der historisierenden Anstrengung nur willkürliche Schranken gesetzt werden können, weil sie nur dort zu einem immer vorläufigen Halt kommt, wo sie sich selbst zum Gegenstand macht. Mit anderen Worten: Die Instrumentarien des kontrollierten Anachronismus sind nicht nur solche, sondern sie sind selbst Gegenstand historischer Analyse. Genau in diesem Sinn geriet seit Ende der 1980er Jahre und zunehmend während der 1990er Jahre das begriffliche Handwerkszeug - sex/gender sowie gender - selbst in den Gegenstandsbereich der Analyse, indem nun die Frage nicht mehr nur die nach historischen Ausprägungen von Geschlechterdifferenz war, sondern auf die Historizität von Geschlechterdifferenz als Operation der Unterscheidung selbst ausgedehnt wurde. ${ }^{41}$

40 Paul Veyne, Die Originalität des Unbekannten. Für eine andere Geschichtsschreibung, Frankfurt a. M. 1988 (Orig. 1976), 7.

4I Oft allerdings wurde und wird diese Frage als solche gleich wieder eingeschränkt durch die Annahme einer grundsärqlichen Omnipräsenz und Omnirelevanz der fraglichen Differenzoperation, die häufig zu einem subsumptionslogischen Vorgehen führt: Welches immer der Gegenstand - es ist immer schon klar, dass „Geschlecht konstruiert wird", und dass dies die jeweils geeignete Fragestellung ist.
Aus welcher Position auch immer sie vorgetragen wurde, handelt es sich dabei um eine radikalisierte Historisierung, die sich auf das eigene konzeptuelle Instrumentarium richtet, das grundsätzlich immer in Gefahr ist zu produzieren, was es voraussetzt beziehungsweise in der Vergangenheit zu finden, was es aus der Gegenwart weiß. ${ }^{42}$ Weil sich in der Kritik an solchem nichts anderes ausdrückt als eine nun reflexiv gewendete genuin historische Anstrengung, waren die Poren in den 1980er und 90er Jahren weit offen für eine ,professionelle Osmose ${ }^{\prime 43}$ von Geschlechtergeschichte und poststrukturalistischen Postulaten nach radikaler De-Essenzialisierung der grundlegenden Kategorien von Wirklichkeitskonstruktion. ${ }^{44}$ Tatsächlich macht es Sinn, wie verschiedentlich argumentiert wurde ${ }^{45}$ den "linguistic turn" als eine radikale Historisierungsanstrengung zu begreifen, die das begriffliche Instrumentarium und die Konstruktion des Gegenstands „Geschlecht", „Geschlechterdifferenz", „Frau“" etc. selbst zu Gegenständen der Analyse macht.

Diese Wendung lässt sich als Korrektur eines unkontrollierten Anachronismus verstehen, der sich aus der Verselbständigung eines kontrollierten Anachronismus - anders formuliert: der Verdinglichung einer analytischen Kategorie zu einem vermeintlichen Sachverhalt - ergeben hat. ${ }^{46}$ Im Durchgang durch solche Arbeit am Begriff wird der kontrollierte Anachronismus (die Risikobereitschaft zu unzeitgemäßen Fragen) im besten Fall zu einem reflexiven Anachronismus (die zusätzliche Risikobereitschaft zur Historisierung des unzeitgemäßen Fragens). Ein solcher schöpft das analytische Potential theoriegeleiteter Geschichtsschreibung aus und begegnet aber der damit verbundenen Gefahr der Verdinglichung analytischer Begriffe sowie derjenigen der theoretischen

42 In meinen Augen treffen sich hier zwei Positionen, die als antagonistisch gehandelt werden: Nämlich die im Rahmen poststrukturalistischer und interpretativ-soziologischer Argumentation formulierte Kritik von Joan Scott an den dem Begriffspaar sex/gender zugrunde liegenden Annahmen einerseits, und die von Bara Duden andererseits, welche die Rede von sex/gender als das Symprom eines spezifisch heurigen, nämlich entkörperten Verständnisses der Person begreift, das als unkontrollierter Anachronismus an die Vergangenheit herangetragen wird, deren Empirisches sich damit nicht verstehen lässt.

43 Carla Hesse, The New Empiricism, in: Cultural and Social History, 1, 2 (2004), 201-207, 201.

44 So war etwa Joan Scotts poststrukturalistisch informierte Kritik am Erfahrungsbegriff keine Negation der Möglichkeit und Wirklichkeit des Subjekts, wie gerne behauptet wird, sondern eine Historisierung von Subjektivirät und Handlungsmacht. Joan W. Scott. The Evidence of Experience, in: torisierung von Subjektivität und Handlungsmacht. Joan W. Scott, The Evidence of Experience,

Terrence McDonald Hg., The Historic Turn in the Human Sciences, Michigan 1996, 319-406.
Vgl. Hesse, Empiricism, wie Anm. 43. Lynn Hunt spricht fast synonym von "historicization “ und

45 Vgl. Hesse, Empiricism, wie Anm. 43. Lynn Hunt spricht fast synonym von "historicization" und Narratives in Gender History, in: Hans Medick u. Anne-Charlott Trepp Hg., Geschlechtergeschichte und Allgemeine Geschichte. Herausforderungen und Perspektiven, Göttingen 1998, 57-97. Ein

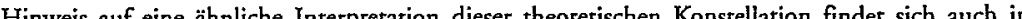
der Cleichsetung von wournant critiqu" und "linguscic turn" bei Wahnich, Anachronisme, wie Anm. 22, 142.

46 Vgl. allerdings zu Verdinglichungstendenzen im Gebrauch der Kategorie Geschlecht selbst in dekonstrukciven Arbeiten: Mary Hawkesworth, Confounding Gender, in: Signs, 22 (1997), 649-685. 
Orthodoxie dadurch, dass die Historizität der Gegenstände und der Analysekategorien bewusst gehalten wird. ${ }^{47}$ Über die Zeit hinweg kann der reflexiv anachronistische Zugriff so einen Gegenstand zunächst konstruieren und isolieren, um ihn dann in jeweils historisch und räumlich spezifischen Kontexten radikal zu historisieren. Dabei muss immer damit gerechnet werden, dass die Einheit des Gegenstandes zerfältt und die Analysekategorie sich als untauglich erweist. Seine heuristische Leistung aber hat der reflexive Anachronismus auch und gerade dann erbracht, ist doch damit nicht nur eine Erkenntnis über Vergangenes gewonnen, die nun als Frage zurück an die Gegenwart getragen werden kann, sondern es ist auch Arbeit am Begriff geleistet.

\section{Narrative Funktion und unerkanntes Wirken der Anachronie}

in der Geschlechtergeschichte

Aus feministischer Perspektive ist die Gegenwart ein Ort der politischen Auseinandersetzung, die einerseits zukunftsgerichtet ist und andererseits das Vergangene zu einem Argument in Aushandlungen über diese Zukunft macht, und zwar als Evidenz (etwa im Sinne des Nachweisens von Strategien der Diskriminierung) oder in einem entnaturalisierenden Sinn (wenn Selbstverständlichkeiten der Gegenwart durch die Rekonstruktion vergangener Transformationen dekonstruiert werden). In beiden Fällen sind Fragen historischer Verlaufsprozesse - von Wandel und Kontinuitär - zentral, da es um Veränderung geht oder mit Veränderbarkeit argumentiert wird. ${ }^{48}$ In deren Erforschung aber ist nicht nur ein Gegenwartsbezug immer im Spiel, weil aus dieser Perspektive keine Epoche in historistischer Haltung „unmittelbar zu Gott" sein kann, sondern es ist auch das Konzept der Anachronie am Werk.

Dieses hat - wenn auch nicht als solches bezeichnet - frauen- und geschlechtergeschichtliche Arbeiten von allem Anfang an informiert: Die Kritik an einer Geschichtsschreibung, die Frauen und die Kategorie Geschlechr ignoriert, sowie das Neuschreiben der Geschichte aus geschlechtergeschichtlicher Perspektive haben die Vorstellung ver-

47 Das scheint mir erwa im Fall der Geschichte der Homosexualität der Fall zu sein, die reflektiert und produkriv mit dem Anachronismus ihres Gegenstands/Namens umgeht.

48 Ich kann hier die Frage nicht aufgreifen, inwiefern im Falle einer dezidiert feministisch ausgerichreten Geschlechtergeschichte die Beschäftigung mit Wandel gleichzeitig konterkariert wird von einem uneingestandenen Begehren nach Kontinuität, ist doch erst durch das Bestehen von Ungleichheitsverhältnissen zwischen den Geschlechtern eine fortdauernde Existenzberechtigung feministischer

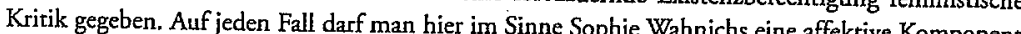
des Gegenwartsbezugs feministischer Geschichtswissenschaft vermuren und zwar ein trackre, richrer sich doch das Begehren gleichermaßen af Ver Brigitre Schneg dis mich in verch gled Brigitte Schnegg, die mich in verschiedenen Gesprächen immer wieder auf diese Ambivalenz auf-
merksam gemachr hat. schiedener Zeitbündel und damit diskontinuierlicher Geschichtlichkeit und heterogener Epochen empirisch ins Werk gesetzt. Die Geschichte der „demokratischen Moderne" als homogen und kontinuierlich vorgestellte zum Beispiel muss anders geschrieben werden, so das Argument, wenn die männliche, weiße und zunächst bürgerliche Gestalt des modernen, mit staatsbürgerlichen Rechten ausgestatteten Subjekts zur Kenntnis genommen und die davon produzierten Ausschlüsse registriert werden: nämlich als die Geschichte einer Hererogenität und Diskontinuität von Aus- und Einschlüssen. Solche Forschungsarbeit mündete denn auch in die Kritik an „allgemeingeschichtlichen" Periodisierungen und in Postulate alternativer Periodisierung. ${ }^{99}$

$\mathrm{Ob}$ allerdings eine Konstellation von unterschiedenen Verlaufsprozessen, wie dic progressive Demokratisierung in der männlichen Bevölkerung und die fortbestehende politische Rechtlosigkeit der weiblichen Bevölkerung im 19. Jahrhundert, in Begriffen verschiedener Zeitbündel und der Anachronie gefasst und gedeutet werden, ist freilich entscheidungsoffen. Anders formuliert: $\mathrm{Ob}$ wir unter Berücksichtigung der Frauen und anderer von politischen Rechten ausgeschlossener Gruppen von einer „verzögerten Demokratisierung" sprechen und damit den Befund in die Gestalt einer rückgreifenden Anachronie fassen (die Frauen des 19. Jahrhunderts leben in politischen Verhältnissen des 18. Jahrhunderts) oder aber mit anderen interpretativen und explanatorischen Implikationen von einem für die Entstehung moderner Demokratien konstitutiven Widerspruch im Gleichzeitigen reden, ist von der reinen Feststellung von Geschlechterund anderen politisch relevanten Ungleichheiten nicht vorentschieden. Umso wichtiger ist es, Deutungen von Anachronien als solche bewusst zu halten.

Mir geht es hier nicht darum, grundsätzlich für oder gegen das Argumentieren in Begriffen der Anachronie zu plädieren. Vielmehr möchte ich die Aufmerksamkeit darauf lenken, dass es sich um eine interpretative und explikative Entscheidung handelt, die aber häufig als solche nicht bewusst ist, weil die Anachronie in stark gegenwartsbezogenen Thematisierungszusammenhängen poetologisch funktioniert (besonders deutlich etwa im Erzählmodus der „Frau, die ihrer Zeit voraus war“, oder, um ein bereits erwähntes Beispiel aufzugreifen, der „Pionierinnenschaft“ weiblicher

49 Vgl. hierzu als klassischer Beitrag: Joan Kelly-Gadol, Did Women Have a Renaissance?, in: Renate Bridenthalt u. Claudia Koonz Hg., Becoming Visible. Women in European History, Boston u. a. 1977, 137-164; vgl. zu Periodisierungsfragen in der Geschlechrergeschichte: Burghartz, Wandel, wie Anm. 11. Hier kommt denn auch die oben formulierre Frage nach der Definirion einer spezifischen Epoche wieder ins Spiel als eine, die deshalb nicht abschließbar ist, weil die in Anschlag gebrachten Kriterien immer für Reinterpretationen offen sind. Dieser Sachverhalt ist auch in die Diskussion um die Notwendigkeit oder aber Unmöglichkeit von historiographischen Metanarrativen eingegangen: vgl. Medick/Trepp, Geschlechrergeschichte, wie Anm. 45, aus hier interessierender Perspektive besonders die Beiträge von Karin Hausen (Die Nicht-Einheit der Geschichte als historiographische Herausforderung. Zur historischen Relevanz und Anstößigkeit der Geschlechtergeschichte, 15-55) und Lynn Hunt (The Challenge of Gender. Deconstruction of Categories and Reconstruction of Narratives in Gender History, 57-97). 
Intellektueller). ${ }^{50}$ Weil Anachronien sich besonders gut eignen, Erzählungen zu organisieren, bleiben die Operationen des damit implizierten und oben erläuterten Verständnisses von Zeit, Wandel und Handlungsmacht unbewusst. ${ }^{\text {sI }}$

Dies ist in zweierlei Hinsicht problematisch: Zum einen verschleiert es der Griff zur Anachronie als Stilmittel, wenn die damit transportierte Erklärung interpretativ zu kurz greift, weil sie nicht als solche wahrgenommen wird. Wenn etwa im Fall der Geschichte moderner Demokratien das Phänomen der für Zeitraum und Gegenstand gerade konstitutiven gleichzeitigen Ein- und Ausschlüsse so lange unbegriffen blieb, wie ausschließlich von einer "Verzögerung" die Rede war. Oder wenn die Feststellung, dass eine „Intellekruelle“ des 16. Jahrhunderts „zu früh kam“ die Erklärung erübrigt, was ihren Handlungsspielraum möglich gemacht hat und damit letztlich kein historisches Wissen erzeugt. Das heißt: Auch ein bewusstes Arbeiten mit Anachronien kann nicht dort aufhören, wo solche konstatiert werden, sondern hat immer nach Erklärungen zu suchen, die gerade die formale Gleichzeitigkeit ernst nehmen - wie eben in der Geschichte moderner Demokratien, wo erst das sich Einlassen auf die Gleichzeitigkeit des Anachronischen die für die Entstehung moderner Demokratien nicht akzidentellen, sondern konstitutiven Widersprüche aufweist. Erst dann ist das Konzept der Anachronie in einem Kracauer'schen Sinn eingelöst: als das Konstatieren von formal gleichzeitigen Sachverhalten, die nichtsdestotrotz miteinander in Zusammenhang stehen. Und erst mit der Klärung dieses Zusammenhangs ist eine Er-Klärung geleistet. Problematisch ist das verborgene Wirken der Anachronie als Konzept deshalb und zum andern insofern, als die Möglichkeiten, welche das darin beschlossene Konzept historischer Zeitlichkeit eröffnet, genau dort nicht ausgeschöpft werden, wo sich das Kerngeschäft der Historikerin befindet: im Feld der theoretischen Konzeptualisierung und empirischen Erforschung von Wandel und Kontinuität beziehungsweise: der temporalen Dynamiken von Geschlechterverhältnissen als historischen Verhältnissen.

\section{Wiedergänger und das Potential der Anachronie}

Von den mittelalterlichen Wiedergängern, also den toten Untoten, wird berichtet, dass sie die Lebenden, die sie heimsuchten, nicht nur fürchterlich erschreckten, sondern

so Anachronien als interprezatives und narratives Instrument scheinen mir besonders dann zum Tragen zu kommen, wenn der historiographische Befund in seiner politischen Relevanz akzentuiert wird was insofern nahe liegt, als die Anachronie eine bevorzugte Figur der politischen Rhetorik ist; vgl. hierzu als konkretes Beispiel Laura S. Strumingher, Looking Back. Women of 1848 and Revolutionary Heritage of 1789, in: Harriet B. Applewhire u. Darline G. Levy Hg WJomen Politics in the Age of the Democratic Revolution, Michigan 1993, 259-285.

I Vgl. zum Sachverhait, dass die explikative Prägekraft narrativer Elemente nichr als solche wahrgenommen wird: Hayden White, Historicism, History, and the Figurative Imagination, in: History and Theory, 14, 4 (1975), 48-67. außerdem mit dem Gewicht ihrer Körper fast erdrückten. Beides zusammen dürfte bei den Heimgesuchten ein Gefühl der ohnmächtigen Bewegungslosigkeit evoziert haben. Es ist dieses Gefühl der Paralyse angesichts der Wiederkehr von etwas Totgeglaubtem, das mich bei zeitgenössischen Phänomenen wie den soziobiologischen Grundierungen der Evolutionary psychology ${ }^{52}$ oder beim medial zum Getöse verstärkten (oder erzeugten) Ruf nach rechtschaffener Weiblichkeit als Rettung anomischer Gesellschaftszustände von Wiedergängern reden lässt. Dabei verschränken sich diese beiden Diskurse mit schöner Regelmäßigkeit - wenn etwa evolutionspsychologische und hirnwissenschaftliche Studien belegen sollen, dass der supponierten Krise demogtaphisch bedrohter und sozial desintegrierter westlicher Industrieländer nur die Frauen beizukommen vermöchten, da sie von Geburt weg mit mehr „sozialer Kompetenz, Einfühlung, Altruismus, Kooperation “ ausgestattet seien als Männer. ${ }^{53}$

Im Empfinden der Historikerin dürfte solche Lähmung allerdings nicht nur dem Erschrecken, sondern überdies einem ermüdenden Moment der Langeweile geschuldet sein: Woher diese Redundanz? Haben Geschlechterforscherinnen solche Argumentationen nicht widerlegt und als herrschaftsstrategische Instrumente zur Errichtung einer ungleichen Geschlechterordnung entlarvt? Erinnern doch solche Verlautbarungen frappant an die in verschiedenen europäischen Ländern um 1900 geführten Anomieund Degenerationsdiskurse, die ebenfalls die Gefahr sinkender Geburtenraten und eine Schwächung sozialer Bande als Folge einer überbordenden Moderne beklagten, der nur mit authentischer Weiblichkeit beizukommen war. ${ }^{54}$ Und Analogien sind nicht nur bei den Inhalten, sondern auch bei möglichen Erklärungen dieser Diskurse zu beobachten: $\mathrm{Zu}$ Beginn des 21. wie des 20. Jahrhunderts handelt es sich um Ordnungsanstrengungen, die auf Gleichberechtigungsforderungen und -dynamiken reagieren. Auch gilt in beiden Fällen, dass diese Ordnungsanstrengungen nicht nur auf Geschlechterverhältnisse, sondern auch auf die nationalen beziehungsweise ethnischen Grenzen im Sinne der "Reinheit" der vermeintlich von Zerfall und Aussterben bedrohten imaginierten Gemeinschaft zielen. Schließlich lässt sich ebenfalls für beide Zeiträume beobachten, dass diese Diskurse auch Bewältigungen zeittypischer Verwerfungen im

52 Das gilt auch für andere sozialwissenschaftliche Felder wie etwa die rational-choice-Soziologie; vgl John Dupré, Human Nature and the Limits of Science, Oxford 2001.

53 Frank Schirrmacher, Minimum. Vom Vergehen und Neuentstehen unserer Gemeinschaft, München 2006, 132, 137. Auch Alain Touraine lässt sich hier zitieren, der zwar nicht biologistisch argumentiert, aber nicht minder naturalisierend ins selbe Horn einer kulturellen und sozialen Zerrissenheit bläst, gegen die es die Frauen in Stellung zu bringen gelte, weil nur sie „das Private mit dem Öffentlichen, den Körper mit dem Geist, die Natur mit der Kultur auszusöhnen und so die Gesellschaft wieder zusammenzusetzen vermögen"; vgl. Le monde des femmes, Paris 2006.

54 Vgl. z. B. Elaine Showalter, Sexual Anarchy. Gender and Culture ar the Fin de Siècle, New York 1990 vgl. außerdem dazu, wie dieser Krisendiskurs die soziologische Zeirdiagnostik als Diskurs über die Moderne in einer Weise prägte, die sich als Traditionsstrang in Touraines Argumentation entäußerr: Barbara L. Marshall u. Anne Witz Hg. Engendering the Social. Feninist Encounters with Sociological Theory, Berkshire/New York 2004. 
sozialstrukturellen Gefüge darstellen: Folgen der Industrialisierung um 1900, neoliberale Prekarisierungen um 2000.

Angesichts der Wiedergängerei solcher Diskurse stellt sich der Historikerin unweigerlich die beunruhigende Frage, ob die Instrumente feministischer Forschung versagr und ihre kritische Kraft verloren haben. ${ }^{55}$ Solche Selbstbefragung ist wichtig, weil sie die Reflexion über Instrumentarien und damit auch deren Umgestaltung vorantreibt und Erstarrung zur Orthodoxie verhindert. Indes ist die Angelegenheit mit Selbstkritik nicht abschließend bewältigt. Dies zu postulieren, hieße, eine grundsätzlich ungebrochene und unmittelbare Wirkungskraft feministischer Kritik anzunehmen, wäre diese nur solid genug ausgeführt. Das Gegenteil kann der Fall sein, wie die perversen Effekte der Freisetzung feministischer Anliegen und Argumente zeigen, die ich weiter oben angesprochen habe. Ich möchte stattdessen vorschlagen, die Wiedergänger aus einer kontrolliert anachronistischen Perspektive in den Blick zu nehmen, um von da aus das Potential der Anachronien und des damit verbundenen Konzepts von Zeit weiter zu konkretisieren.

Nicole Loraux weist in ihrer Verteidigung des (kontrollierten) Anachronismus darauf hin, „dass es Sinn macht, den Phänomenen der Repetition innerhalb der chronologischen Zeit der Geschichte Aufmerksamkeit zu schenken“. ${ }^{\circ 6}$ Tatsächlich handelt es sich beim geschilderten Wiedergänger um die Repetition eines Sachverhaltes, der Historikerinnen wohl vertraut ist: Dass Ordnungen der Gesellschaft sich über Ordnungen der Geschlechter organisieren, und dass diese Ordnungen wiederum verknüpft sind mi solchen, die entlang ethnischer, nationaler oder anderer Kategorien eine imaginierte Gemeinschaft vorstellen. Doch muss der Begriff „Repetition“ ernst genommen werden Die Einheiten einer Repetition sind nie identisch, denn die Repetition als Akt oder Vorgang ereignet sich in einer raumzeitlichen Dimension. Repetiert wird in einem immer differenten Kontext, wie minimal die Differenz auch sein mag. In jedem Fall vergeht Zeit und zwar eben nicht nur im Sinne des Metrums der chronologischen Zeit, vielmehr vergehen darüber hinaus Konstellationen von Zeitbündeln, die sich dabei verändern. Mit anderen Worten: Repetition erzeugt nicht das Immergleiche. Und wenn weiter davon ausgegangen wird, dass Kontinuität durch Repetition zustande kommt dann ist der Kontinuität Diskontinuität immer schon inhärent.

So muss ein erster Blick, der Analogien zwischen den Krisendiskursen 1900 und 2000 feststellt, in einem zweiten Schritt und im Sinne des Loraux'schen Hin und Her die nötigen Unterscheidungen als Diskontinuitäten im Kontinuierlichen eruieren. So lässt sich etwa die Frage stellen, ob die analogen Ordnungsanstrengungen auf unterschiedlich geartete Umbrüche reagieren: um 2000 auf solche im Strukturellen, um 1900

55 Vgl. Joan W. Scott, Millenial Fantasies. The Future of ,Gender' in the 21st Century, in: Claudia Honegger u. Caroline Arni Hg., Gender - die Tücken einer Karegorie. Joan W. Scott, Geschichre und Politik, Zürich 2001, 19-37. auf solche in der Praxis. Verlagert sich die Herstellung von Geschlechterdifferenz und Geschlechterungleichheit gegenwärtig in dem Maße in Praktiken und Interaktionen, in dem auf institutioneller Ebene formale Gleichheit statuiert wird, ${ }^{57}$ so waren um 1900 die formalen Strukturen (des Eherechts, der Berufsausbildung, des Politischen etc.) enger gefasst hinsichtlich der Platzanweisungen an die Geschlechter, während sich in Praktiken und weiblichen Identitätsentwürfen eine Vielfalt feststellen lässt, die diese Platzanweisungen negiert und ignoriert. Trifft diese Beobachtung zu, so haben wir es mit Anachronien im Sinne von gegenläufigen Entwicklungen zweier verschiedener Dimensionen sozialer Wirklichkeit zu tun, die aber in ihren Dynamiken aufeinander reagieren.

An diesem Beispiel zeigr sich, dass eine Analyse der oben angesprochenen Widersprüchlichkeiten in den aktuellen Geschlechterverhältnissen (formal-rechtliche Gleichstellung, praktische Ungleichheit) ohne historische Reflexion nicht auskommt, wie umgekehrt die Erklärung historisch spezifischer Verhältnisse ohne den Blick auf ein Vorher oder Nachher nicht genügt. In so orientierten Untersuchungen ließe sich empirisch präziser belegen und theoretisch stringenter begründen, womit häufig auf eher intuitiv gefühlter, denn argumentativ begründeter Basis (und im Übrigen in präsentistischer ebenso wie in historisierender Verkürzung) hantiert wird: dass die eine oder andere Dimension des Sozialen „besonders zählebig" sei, wobei diese eine besonders veränderungsresistente Dimension in der ersten Untersuchung ein kulturelles Deutungsmuster, in der zweiten eine sozioökonomische Struktur und in der dritten ein Identitätsmuster ist. Und es ließen sich solche Fragen eher als empirisch offene nach jeweiligen Konstellationen und als Aufforderungen zur präzisen historischen Rekonstruktion fassen, denn in Begriffen einer spontantheoretischen a priori Hierarchie von gestuften Zäblebigkeiten. Die Aufmerksamkeit wäre dann auf die unterschiedlichen Zeitlichkeiten und ,Geschichten ' der Dimensionen gerichtet, die „Geschlecht" ausmachen. Kulturelle Deutungen, normative Konzepte, institutionelle Organisation und subjektive Identität bewegen sich in unterschiedlichen Rhythmen, die aber je gleichzeitig anwesend sind und aber auch je nach historisch spezifischer Gesellschaftsformation unterschiedlich ins Gewicht fallen. ${ }^{58}$ Weiter lassen sich diese Kategorien intern nach verschiedenen Temporalitäten differenzieren. So birgt etwa subjektive Identität in sich bereits eine doppelte Zeitlichkeit: die biographische Zeit des Subjekts und die Zeit epochaler Subjektivierungsformen. Entsprechend hätten sich empirische Untersuchungen immer mit der Rekonstruktion der temporalen Logiken und Strukturen der gegenständlichen Einheit und des sie konstituierenden Kontextes zu beschäftigen.

57 Bettina Heintz u. Eva Nadai, Geschlecht und Kontext. De-Institutionalisierungsprozesse und geschlechtliche Differenzierung, in: Zeitschrift für Soziologie, 27, 2 (1998), 75-93.

58 Ich arbeite hier mit den Dimensionen nach Joan W. Scott, Gender: A Useful Caregory of Historical Analysis, in: American Historical Review, 91, 5 (1986), 1053-1075. Selbstverständlich sind auch andere kategoriale Systematiken denkbar 


\section{Zum Schluss: „Das Rätsel der Zeit”}

Ich habe die Themarik des Anachronismus und der Anachronien hier nicht nur deshalb aufgegriffen, weil es mir lohnenswert scheint, die Transformationen und das theoretische Potenzial der Geschlechtergeschichte entlang einer genuin geschichtstheoretischen Problematik durchzudenken. Ich habe sie auch deshalb aufgegriffen, weil sie zur Reflexion des Gegenwartsbezuges historischer Arbeit veranlasst. Die Produktion von Wissen über die Vergangenheit habe, so Joan Scott, in der Geschichte des Feminismus immer dazu gedient, die Gewissheiten der Gegenwart aufzubrechen und so den Weg freizumachen für die Vorstellung einer anderen Zukunft. ${ }^{59} \mathrm{Im}$ Zusammenhang meiner Überlegungen lässt sich dies auch umgekehrt formulieren: Es geht um einen analytischen Bezug zur Gegenwart als Voraussetzung für historisches Denken. Wenn, wie Sophie Wahnich argumentiert, Aktualität historische Arbeit organisiert, indem sie bei der Historikerin Affekte erzeugt, dann gilt es diese zu reflektieren. Mit den Widersprüchen in den Geschlechterverhältnissen, den perversen Effekten eines zirkulierenden feministischen Diskurses und den Wiedergängern habe ich Phänomene angesprochen, in deren Kontext sich das Verhälnnis von Gegenwart und Geschlechtergeschichte heute in spezifischen Weise knüpft: in einer, die eben den Affekt der Lähmung im Gegenwartsbezug der Geschlechtergeschichte erzeugt. Ein gelähmter Gegenwartsbezug aber blockiert historisches Denken.

Das ist insofern nicht harmlos, als es in eben dieser Gegenwart eine Konkurrenz auf dem Gebiet des Geschichtlichen gibt, die über verschiedene Deutungen des Vergangenen hinausgeht. Obschon ich das so präsentiert habe, scheint mir nicht, dass die evolutionspsychologischen Letztbegründungen reaktionärer Anomiediskurse noch in dem Sinne biologistische sind, wie Biologismus im feministischen Diskurs gemeinhin verstanden wird, nämlich als Rekurs auf eine unveränderliche, ahistorische Natur. Der Biologismus der Evolutionspsychologie argumentiert weniger mit anatomischen Strukturen als vielmehr mit urzeitlichen Verhälnnissen und also in gewissem Sinne historisch. Die Natur der Evolutionspsychologie und sämtlicher weiterer Vertreter neopositivistischer Verhaltenswissenschaften ist eine historische, eine wandelbare Natur, deren wenn auch extrem langsam arbeitendes - Bewegungsgesetz die Entstehung von Regeln ist, die das menschliche Zusammenleben zunehmend ,optimal' hinsichtlich des Überlebens der Gattung organisieren und auf die alle Komplexität reduziert wird. ${ }^{60}$ Dies sind Hinweise darauf, dass die Konkurrenz der Deutungen von Welt gegenwärtig gerade

59 Joan W. Scott, Feminism's History, in: Journal of Women's History, 16, 2 (2004), 10-29, 18.

60 Dupré, Nature, wie Anm. 52. Freilich kann diese Beobachtung im Sinn des Loraux'schen Hin und Her als Frage an die Vergangenheit gerichtet werden; es könnte sich weisen, dass Natur in der Moderne nie derart eindeutig, wie es die sozialwissenschaftliche Argumentation in den 1960/70ern erwa in der Begrifflichkeit von sex/gender voraussetzt, als starisch einer wandelbaren Kultur entgegengesetzt wurde. auch hinsichtlich von Geschlechterdifferenz und Geschlechterverhältnissen längst nicht mehr eine zwischen sozial- und kulturwissenschaftlichen einerseits und naturwissenschaftlichen andererseits ist, sondern eine um unterschiedliche Deutungen des Historischen und dessen temporale Dynamik. Das aber heißt, dass die Geschlechtergeschichte - und nicht nur sie - mit Konkurrenz nicht außerhalb des, sondern auf dem Gebiete der historisierenden Erklärung konfrontiert ist, die sich auch unterscheidet von derjenigen innerhalb des historischen Feldes.

Anschließend an diese Überlegungen lässt sich vielleicht konturieren, was das Weitere ist, das sich im Unbehagen an der eingangs konstatierten Ratlosigkeit dokumentiert: die - bewusste oder unbewusste - Wahrnehmung einer Konkurrenz auf dem Gebiet des Historischen, die Sehnsüchte nach einem konzeptuellen Fluchtpunkt weckt, den es in einer ausdifferenzierten und normalisierten Geschlechtergeschichte nicht mehr geben kann. Diese gewissermaßen disziplinenpolitische Problematik hat einen gesellschaftspolitischen Resonanzraum, aber sie ist nicht als politisches Problem anzugehen, sondern auf dem Weg geschichtstheoretisch informierter Analyse von Temporalitäten. In einer Gegenwart, die nicht nur, aber besonders heftig was die Geschlechterverhältnisse betrifft, derart intensiv mit der Frage beschäftigt ist, was sich verändert und was sich gleich bleibt, in einer Gegenwart, die in ihren Selbstvergewisserungsdiskursen derart viel historische Mythen und Amnesien hervorbringt und wiederbelebt (das christliche Abendland, die traditionelle Familienstruktur), in einer Gegenwart, die indem sie geschichtsvergessen ist, doch obsessiv mit Geschichtlichkeit beschäftigt ist, besteht eine erneute - Herausforderung darin, die Zeit und ihre Rhythmen, die temporalen Konstellationen in Vergangenheit und Gegenwart, aber auch die Geschichtlichkeit des Denkens von Zeit zu reflektieren.

Für die Geschlechtergeschichte stellen sich in diesem Kontext zwei Herausforderungen. Die erste, selbstbezügliche, besteht darin, die Transformationen der Geschlechtergeschichte in geschichtstheoretischen Begriffen zu reflektieren: Mit welchen Zeitkonzepten wurde und wird in der Geschlechtergeschichte eigentlich gearbeitet? Welche Annahmen temporaler Logik informieren die Arbeiten von Geschlechterhistorikerinnen? Und wie ist der Gegenwartsbezug der Geschlechtergeschichte beschaffen? Dass solche Fragen - konkret zum Beispiel Periodisierungsdiskussionen - in den Hintergrund getreten sind, scheint mir wiederum mit Spezialisierungstendenzen und einem damit zusammenhängenden vernachlässigten innerdisziplinären Dialog über Epochengrenzen hinweg zusammenzuhängen. Was aber nicht Anlass sein sollte, sich nostalgischer Trauer über eine verloren gegangene Einheit hinzugeben, sondern die Anerkennung der Heterogenität des Feldes zum Anlass für Zusammenarbeit zu machen.

Die zweite Herausforderung konfrontiert Geschlechterhistorikerinnen mir der Notwendigkeit, sich Zumutungen - einerlei ob von ,Außen' herangetragen oder vom eigenen Begehren angetrieben - zu widersetzen, die sich auf ihr Kerngeschäft richten, dieses aber in einer Weise zurichten, die es negiert: indem formelfähige Zeitdiagnosen im Sinne einer abschließenden oder schon nur einer Zwischenbilanz in der Geschichte 
der Geschlechterverhältnisse und -differenz eingefordert werden, die nur auf der Grundlage der Fiktion einer homogenen Gegenwartsepoche zu haben sind. ${ }^{61}$ Stattdessen könnte es sich die Geschlechtergeschichte zum Anliegen machen, danach zu fragen, wie bestimmte Prozesse, Ereignisse, Verhältnisse und Zustände eingefügt sind in Zeitreihen und heterogene temporale Strukturen und Rhythmen. ${ }^{62}$ Dabei gälte es ein Dilemma auszuhalten, das nicht einfach ein zeitdiagnostisches ist, sondern ein geschichtstheoretisches des Denkens der Zeit, ja, in den Begriffen Siegfried Kracauer, „das Rätsel der Zeit" schlechthin: Die Tatsache, dass wir es mit einer „inkohärenten Reihe geformter Zeiten" und der "chronologischen Zeit als homogenen Fluss" zugleich zu tun haben. ${ }^{63}$

Darin könnte auch ein spezifischer Beitrag bestehen, den Geschlechterhistorikerinnen zum Verständnis der aktuellen Geschlechterverhältnisse leisten können. Und damit ist nicht gemeint, ,Spuren' der Antike, des Mitrelalters und der Neuzeit in der Gegenwart aufzudecken (das wäre das Geschäft politischer Rhetorik oder musealer Präsentationslogik), sondern die verschiedenen Zeitlichkeiten zu einem gegebenen Zeitpunkt zu rekonstruieren und die Gegenwart und die Vergangenheit einander zur Frage zu machen. Solches aber lässt sich aus der Position eines gelähmten Gegenwartsbezugs nicht bewerkstelligen, weil darin der "heuristische Wert der Gegenwart" (la valeur heuristique du présent) $)^{64}$ verfällt. Der Lähmung wäre so gesehen nicht die Sehnsucht nach programmatisch verbindlichen Fluchtpunkten entgegenzusetzen, sondern die Reflexion auf das, was das eigene Denken und Arbeiten konstituiert: der Gegenwartsbezug und die vergehende Zeit.

61 Und die außerdem häuffig in die eine oder andere Richtung linear ausgerichter sind: meist teleologisch auf das "modern is better" (vgl. Hunt, Challenge, wie Anm. 45, 83), gelegentlich aber auch nostalgisch auf eine ,bessere ${ }^{c}$ Vergangenheit angesichts gegenwärtiger Verwerfungen eines angenommenen "backlash".

62 Vgl. dazu auch Hunt, Challenge, wie Anm. 45, 89-92, die auf dieser Grundlage neue Metanarrarive infordert.

63 Kracauer, Geschichte, wie Anm. 30, 153

64 François Dosse, De l'usage raisonné de l'anachronisme, in: CLIO \& EspacesTemps, 87-88 (2004), $156-171,157$. 